\title{
Identical twins with uterus didelphys and duplex kidneys
}

\author{
E. Daw and P. Toon \\ North Manchester General Hospital, Crumpsall, Manchester, UK.
}

Summary: Identical twins with uterus didelphys and bilateral duplex kidneys are reported.

\section{Introduction}

Congenital uterine abnormalities occur as a result of partial or complete failure of Mullerian duct fusion probably about the fifth or sixth week of fetal life. Maternal and/or placental factors have been implicated. There are a few reports of a double uterus occurring in sisters and their mothers (Polishuk \& Ron, 1974; Prucha, 1983) which suggest a dominant autosomal gene as a possible genetic cause. Renal tract abnormalities are associated with uterine abnormalities (Eisenberg et al., 1982) and we wish to report identical twins with uterus didelphys and bilateral duplex kidneys.

\section{Case histories}

The first patient was referred to the gynaecological clinic at the age of twenty-four with a two year history of infertility. On examination the patient was found to have two cervices with a vaginal septum between them. At examination under anaesthesia and dilatation and curettage she was found to have two uteri with no communication between the cavities. Post-operatively the patient was informed of the findings and possible problems that could occur during pregnancy. In view of the known association of uterine abnormalities with renal tract abnormalities an intravenous pyelogram was performed. This showed double kidneys on each side, with a double collecting system and double ureter throughout the whole length (Figure 1).

Her unmarried identical twin sister was then referred to the gynaecological clinic as she was worried by the findings in her twin and wished reassurance. Examination, both in the out-patient department and under anaesthesia with dilatation and curettage, showed her to have a vaginal septum, two cervices and double uteri without communication between the cavities. An intravenous pyelogram showed double

E. Daw M.B., Ch.B., F.R.C.O.G.: P. Toon M.B., Ch.B., M.R.C.O.G.

Correspondence: E. Daw.

Accepted: 8 May 1984 kidneys on each side, with double collecting systems and double ureters, similar to her sister. The sisters were confirmed as identical twins by the presence of five identical blood groups on testing their sera. Neither twin had any history of symptoms of renal tract disease.

\section{Discussion}

There are many reports in the literature of partial or complete failure of Mullerian duct fusion, the women being otherwise perfectly normal. The familial nature of this condition may be more frequent than is recognised as the same gynaecologist rarely attends two or more generations of women of the same family (Prucha, 1983). The cases reported here would confirm a possible genetic cause. However Heinonen et al. (1982) reviewed 182 women with uterine abnormalities seen over a period of 18 years, without commenting on any familial incidence.

Associated urinary tract developmental defects are recorded in association with uterine abnormalities (Loendersloop, 1977) although Polishuk \& Ron (1974) did not record an associated urinary tract abnormality in three families. In the series of Heinonon et al. (1982) only 53 out of 182 patients had intravenous pyelography. In 12 cases $(23 \%)$ an abnormality was found: 6 patients had an absent kidney, 2 a horseshoe kidney, 1 a pelvic kidney and 3 a duplication of the collecting system. The greatest incidence of urinary tract abnormality was associated with unicornuate uterus.

Uterine anomalies are associated with obstetric complications such as habitual abortion, premature rupture of the membranes, premature delivery, breech presentation and a higher perinatal mortality (Heinonon et al., 1982). Prior knowledge of the existence of the uterine anomaly has important implications for antenatal care and management of the patient, such that the existence of a uterine anomaly in a family merits investigation of other females in the family. 


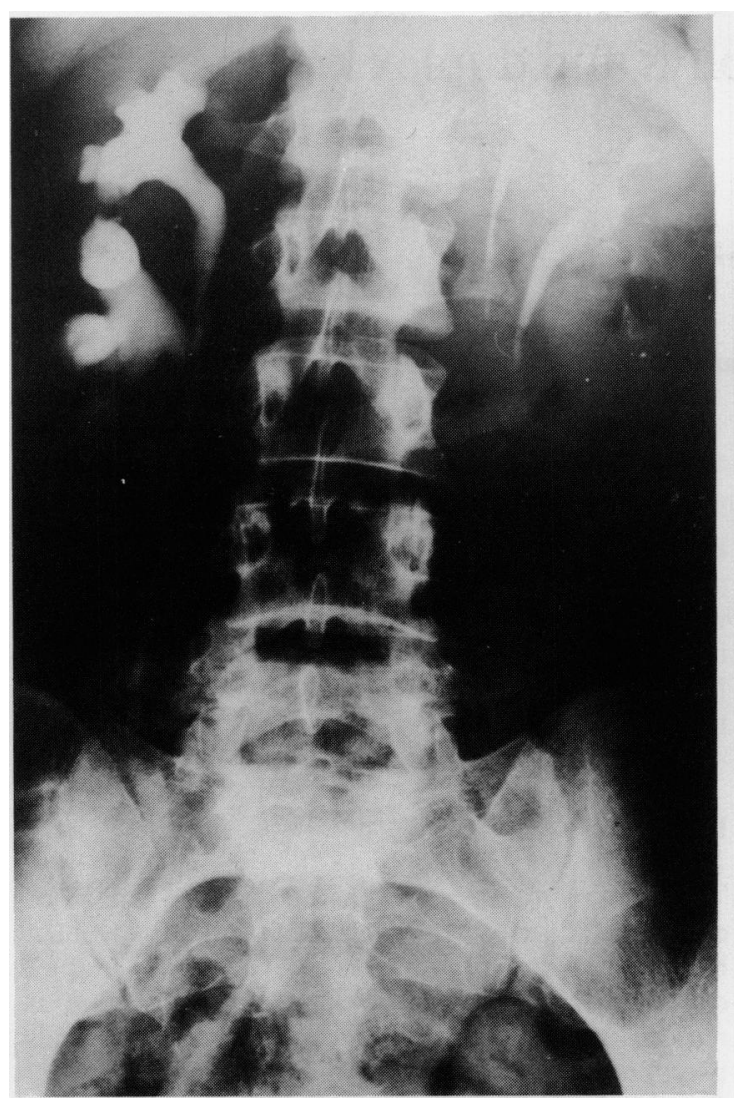

Twin 1;

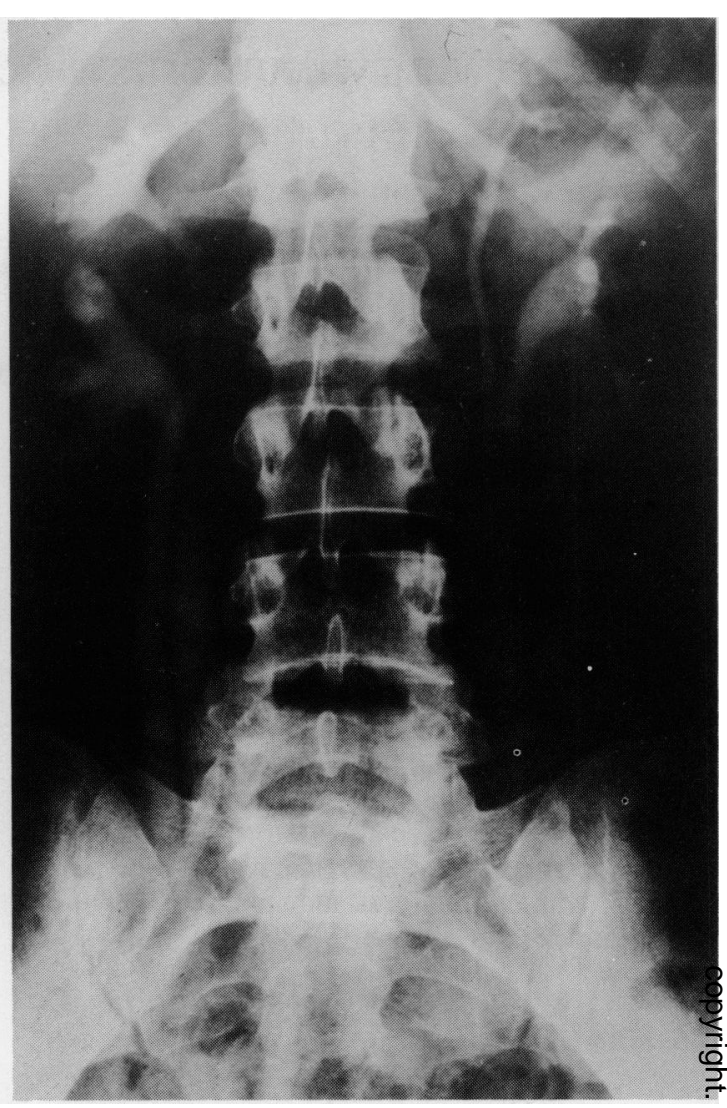

Twin 2.

Figure 1 Intravenous pyelograms in twin sisters with uterus didelphys.

\section{References}

EISENBERG, E., FARBER, M., MITCHELL, G.W., TURKSOY, R.N. \& RULE, A.H. (1982). Complete duplication of the uterus and cervix with a unilateral imperforate vagina. Obstetrics and Gynecology, 60, 259.

HEINONEN, P.K., SAARIKOWSKI, S. \& PYSTYNAN, P. (1982). Reproductive performance of women with uterine anomalies, an evaluation of 182 cases. Acta Obstetrica et Gynecologica Scandinavica, 61, 157.
LOENDERSLOOP, E.W. (1977). Twin pregnancy in double uterus. American Journal of Obstetrics and Gynecology. $127,682$.

POLISHUK, W.Z. \& RON, N.A. (1974). Familial bicornuate and double uterus. American Journal of Obstetrics and Gynecology. 119, 982.

PRUCHA, Z. (1983). Double trouble. World Medicine,18, 62. 